\title{
Preliminary comments on proteasome inhibition and cardiovascular disease
}

\author{
Friedrich C. Luft
}

Received: 27 May 2009/Accepted: 27 May 2009/Published online: 18 June 2009

(C) Springer-Verlag 2009

Proteasomes are large protein complexes inside all eukaryotic cells and some bacteria. The proteasome functions to degrade unneeded or damaged proteins by proteolysis, a chemical reaction that breaks peptide bonds. Enzymes that carry out such reactions are called proteases. Proteasomes are part of a major mechanism by which cells regulate the concentration of particular proteins and degrade misfolded proteins. The degradation process yields peptides of about seven to eight amino acids long, which can then be further degraded into amino acids and used in synthesizing new proteins. Proteins are tagged for degradation by a small protein called ubiquitin. Enzymes called ubiquitin ligases catalyze the tagging reaction. Once a protein is tagged with a single ubiquitin molecule, the tagging is a signal to other ligases to attach additional ubiquitin molecules. The result is a polyubiquitin chain that is bound by the proteasome, allowing it to degrade the tagged protein.

The proteasome is built as a large barrel-like complex containing a "core" of four stacked rings around a central pore. Each ring is composed of seven individual proteins. The inner two rings are made of seven $\beta$ subunits that contain the six protease active sites. These sites are located on the interior surface of the rings, so that the target protein must enter the central pore before it is degraded. The outer two rings each contain seven $\alpha$ subunits whose function is to maintain a "gate" through which proteins enter the barrel. Binding to "cap" structures controls these $\alpha$ subunits or regulatory particles that recognize polyubiquitin tags attached to protein substrates and initiate the degradation

F. C. Luft $(\bowtie)$

Experimental and Clinical Research Center, Franz-Volhard Clinic, Berlin, Germany

e-mail: luft@charite.de process. The overall system of ubiquitination and proteasomal degradation is known as the ubiquitin-proteasome system. The proteasomal degradation pathway is essential for many cellular processes, including the cell cycle, the regulation of gene expression, and responses to oxidative stress. The importance of proteolytic degradation inside cells and the role of ubiquitin in proteolytic pathways was a Nobel Prize-winning event in 2004.

Proteasome inhibitors can block the proteasome pathway. Disulfiram, an anti-alcohol drug inhibiting alcohol dehydrogenase and epigallocatechin-3 gallate, an agent in "green tea," which therefore must be good for you, have been identified as possible proteasome inhibitors. However, bortezomib is the first drug developed for this purpose; the compound has been used therapeutically since 2003 [1]. The boron atom in bortezomib binds the catalytic site of the $26 \mathrm{~S}$ proteasome with high affinity and specificity. In normal cells, the proteasome regulates protein expression and function by degradation of ubiquitinylated proteins and also cleanses the cell of abnormal or misfolded proteins. Clinical and preclinical data support a role for proteasomes in maintaining the immortal phenotype of myeloma cells, and cell-culture and xenograft data support a similar function in solid tumors. While multiple mechanisms are likely to be involved, proteasome inhibition may prevent degradation of pro-apoptotic factors, permitting activation of programmed cell death in neoplastic cells dependent upon suppression of pro-apoptotic pathways. Bortezomib has become a backbone in the treatment of refractory multiple myeloma. However, perhaps bortezomib might have a place in the treatment of other B-cell-related diseases.

Long-lived plasma cells producing autoantibodies resist current therapeutic and experimental approaches. Recently, Neubert et al. [2] showed that the sensitivity of myeloma 
cells toward proteasome inhibitors directly correlates with their immunoglobulin synthesis rates. Therefore, they hypothesized that normal plasma cells are also hypersensitive to proteasome inhibition owing to their extremely high amount of protein biosynthesis. The same investigators next showed that bortezomib eliminates both short- and longlived plasma cells by activation of the terminal unfolded protein response [2]. The disease they addressed in an animal model is systemic lupus erythematosus, a generalized autoimmune model disease in humans and experimental animals. Treatment with bortezomib-depleted plasma cells producing antibodies against double-stranded DNA eliminated autoantibody production, ameliorated glomerulonephritis, and prolonged survival of two mouse strains with a lupus erythematosus-like disease. The authors suggested that the elimination of autoreactive plasma cells by proteasome inhibitors might represent a new treatment strategy for antibody-mediated diseases.

The proteasome is required for both of the major pathways leading to nuclear factor-kappa B (NF-KB) activation [1]. NF$\mathrm{KB}$ is a key transcription factor mediating defense, inflammation, and innate immunity present in the sea anemone and all that came after this organism. NF-KB is carefully regulated in the cytoplasm to keep the transcription factor from going off "too soon." The notion of proteasome investigators was that the proteasome mediates the IKB kinase-stimulated degradation of NF-kB's physiological inhibitor, I $\mathrm{I} B \alpha$. I $\mathrm{I} B \alpha$ is a protein that interacts with NFKB's nuclear localization signal to prevent nuclear import of the transcription factor, and it is also required for proteolytic processing of the p100 precursor of p52 in the noncanonical pathway (Fig. 1). These effects gained the attention of cancer researchers, particularly as numerous cancers exhibit innate NF- $\mathrm{KB}$ activation. Thus, we come full circle, namely B-cell regulation and NF- $\mathrm{KB}$ control.

$\mathrm{NF}-\mathrm{KB}$ is involved in ubiquitous inflammatory processes that impact directly on cardiovascular disease. Herrmann et al. [3] tested the possibility that proteasome inhibition might be of value in a model of atherosclerosis. This disease features B-cell disturbances, apoptosis-related mechanisms, oxidative stress, and NF- $\mathrm{KB}$ activation. The investigators reasoned that the proteasome could be responsible for the degradation of oxidized proteins. However, proteasome inhibition has also been shown to generate oxidative stress in vitro. The authors randomized young pigs to a normal or high-cholesterol diet without or with twice weekly subcutaneous injections of a proteasome inhibitor. They tested coronary vascular function and structure. They found that chronic proteasome inhibition was associated with increased coronary artery oxidative stress and early atherosclerosis. Their findings supported the significance of the proteasome and related protein quality control for vascular biology and pathology but

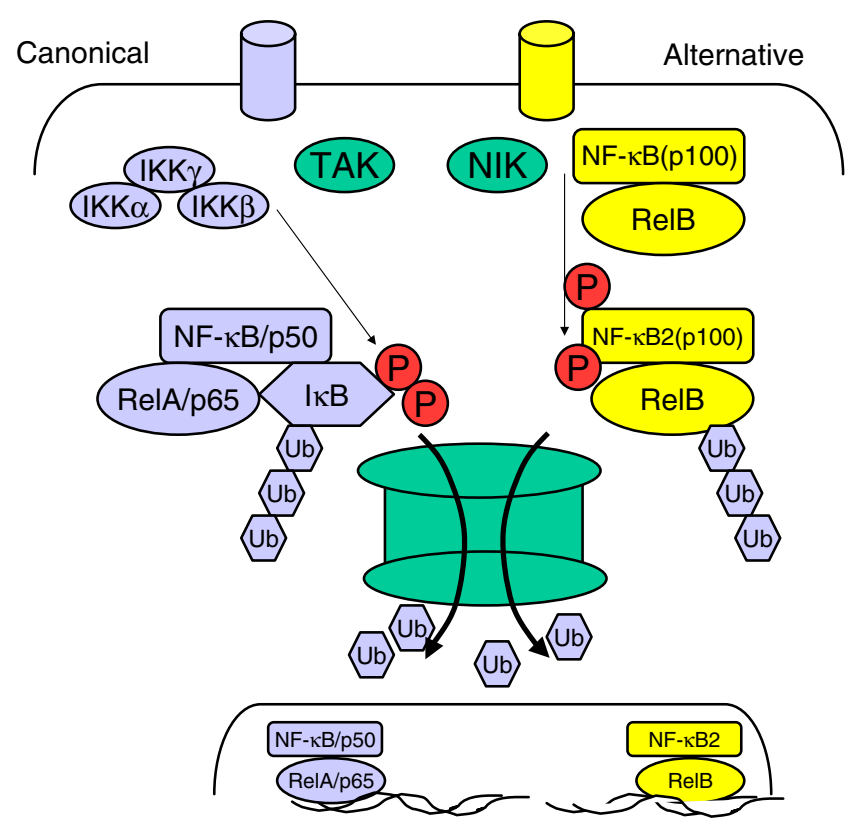

Fig. 1 The proteasome (green barrel) influences both pathways to NF-kB activation. The "canonical" pathway focuses on NF-kB's inhibitor, I $\mathrm{K} B \alpha$, a proteasome substrate. When phosphorylated, the inhibitor is cleaved, ubiquitinated, and degraded. The "alternative" pathway involves the NF- $\mathrm{kB}$-inducing kinase (NIK). NIK phosphorylates a precursor, leading to its ubiquitination, subsequent partial degradation, and activation by the proteasome. Proteasome inhibitors can interfere with both processes. The figure was adapted from [1]

raised the suggestion that proteasome inhibition could contribute to coronary atherosclerosis. The accompanying editorialist to this paper raised several questions [4]. What are the molecular mechanisms for the increase in endogenous oxidative stress by proteasome inhibitor? Does ubiquitin-proteasome system play a role in regulating tetrahydrobiopterin (BHR) bioavailability, thereby controlling eNOS activity and endothelial function? Is effect of proteasome inhibitor on atherosclerotic lesion dosedependent (i.e., reduce or increase neointimal formation)? Is enhancement of proteasome activity beneficial for reducing atherosclerosis?

The questions raised by Fukai remain unanswered [4]. However, in this issue, we have another vascular study looking at bortezomib. Ludwig et al. [5] performed in vitro studies in human umbilical-vein endothelial cells (HUVEC). The production of reactive oxygen was diminished by treatment. The authors also found that bortezomib lowered the adhesion of monocytes to the HUVEC. Inhibition of NF-kB, however, was incomplete. When Dahl salt-sensitive rats were fed high salt, the treatment with bortezomib reduced blood pressure and vascular cell adhesion molecule 1 expression in the aorta compared to control systems. As with all animal systems, there are problems. The authors did not measure blood pressure with 
telemetry. Thus, we can only be moderately reassured that the effects were independent of blood pressure. The expected NF-KB activation and its inhibition with bortezomib could not be convincingly replicated in the cells. In the rats, electrophoretic mobility shift assays for NF-KB activation in blood vessels, with or without bortezomib, would have been interesting. Perhaps, the authors are correct in terms of their negative NF- $\mathrm{kB}$ results. However, there are other mechanisms by which proteasome inhibitors could function. These mechanisms include stabilization of pro-apoptotic proteins by influencing p53 and the BCL-2 family proteins. Cell death may also occur via activation of the terminal unfolded protein response. The salubrious in vitro and in vivo results are not mechanistically explained. Thus, we carry away only half a loaf. Do proteasome inhibitors show any promise in cardiovascular or immune disease? Is this pathway worthwhile pursuing further? The current study answers none of these questions definitively. Needed here is a comprehensive program using several different models and lots of innovative cell biology and molecular pharmacology. The notion is tantalizing but not yet proved.

Respectfully,

Friedrich C. Luft

\section{References}

1. McConkey DJ, Zhu K (2008) Mechanisms of proteasome inhibitor action and resistance in cancer. Drug Resist Updat 11:164-179

2. Neubert K, Meister S, Moser K, Weisel F, Maseda D, Amann K, Wiethe C, Winkler TH, Kalden JR, Manz RA, Voll RE (2008) The proteasome inhibitor bortezomib depletes plasma cells and protects mice with lupus-like disease from nephritis. Nat Med 14:748-755

3. Herrmann J, Saguner AM, Versari D, Peterson TE, Chade A, Olson M, Lerman LO, Lerman A (2007) Chronic proteasome inhibition contributes to coronary atherosclerosis. Circ Res 101:865-874

4. Fukai T (2007) Targeting proteasome worsens atherosclerosis. Circ Res 101:859-861

5. Ludwig A, Fechner M, Wilck N, Meiners S, Grimbo N, Baumann G, Stangl V, Stangl K (2009) Potent anti-inflammatory effects of low-dose proteasome inhibition in the vascular system. J Mol Med (this issue) 